Revue d'histoire de l'Amérique française

REVUE D.HISTOIRE DE L'AMÉRIQUE FRANÇAISE

\title{
Les cercles des jeunes naturalistes, ampleur et nature du mouvement, 1931-1971
}

\section{Pierrick Malissard}

Volume 50, numéro 1, été 1996

URI : https://id.erudit.org/iderudit/305487ar

DOI : https://doi.org/10.7202/305487ar

Aller au sommaire du numéro

\section{Éditeur(s)}

Institut d'histoire de l'Amérique française

ISSN

0035-2357 (imprimé)

1492-1383 (numérique)

Découvrir la revue

Citer cet article

Malissard, P. (1996). Les cercles des jeunes naturalistes, ampleur et nature du mouvement, 1931-1971. Revue d'histoire de l'Amérique française, 50(1), 3-27. https://doi.org/10.7202/305487ar
Résumé de l'article

Cet article présente une analyse détaillée du mouvement des Cercles des jeunes naturalistes (CJN), de sa naissance jusqu'au début des années 1970. S'appuyant sur le traitement informatique d'une partie des archives des Cercles, cette analyse précise l'image des CJN, notamment en montrant l'importance considérable des congrégations religieuses féminines dans le mouvement. Cette étude tend également à nuancer le rapport entre les milieux scientifiques canadiens français et les Cercles : les membres des CJN, entre autres parce qu'ils sont trop jeunes, ne peuvent vraisemblablement pas être assimilés au mouvement scientifique qui commence à s'affirmer au Québec dans les années 1920-1930. 


\title{
LES CERCLES DES JEUNES NATURALISTES AMPLEUR ET NATURE DU MOUVEMENT, 1931-1971*
}

\author{
PIERRICK MALISSARD \\ Département d'histoire \\ Université du Québec à Montréal
}

\section{RÉSUMÉ}

Cet article présente une analyse détaillée du mouvement des Cercles des jeunes naturalistes (CJN), de sa naissance jusqu'au début des années 1970. S'appuyant sur le traitement informatique d'une partie des archives des Cercles, cette analyse précise l'image des CJN, notamment en montrant l'importance considérable des congrégations religieuses féminines dans le mouvement. Cette étude tend également à nuancer le rapport entre les milieux scientifiques canadiens français et les Cercles: les membres des CJN, entre autres parce qu'ils sont trop jeunes, ne peuvent vraisemblablement pas être assimilés au mouvement scientifique qui commence à s'affirmer au Québec dans les années 1920-1930.

\begin{abstract}
This paper examines the history of the Junior naturalists Clubs movement from its birth to the beginning of the 1970s. Drawing upon a computer analysis of part of the Clubs' archives, the study presents a more precise image of the movement, emphasizing the importance of women's religious congregations within it. Furthermore, the paper argues for a more balanced view of the relationship between the FrenchCanadian scientific community and the Clubs: the Clubs' members are likely not to be assimilated to the scientistic movement, in part because most of them were too young.
\end{abstract}

* Cet article doit beaucoup aux conseils et aux commentaires de Camille Limoges et Yves Gingras. Nous tenons à les remercier. 


\section{INTRODUCTION}

Le rôle fondamental que les sciences jouent dans les sociétés contemporaines a grandement stimulé, au Québec comme ailleurs, la publication de travaux sur la dimension historique du développement scientifique. Il reste que même des questions assez souvent examinées, telles les conditions d'émergence de l'espace scientifique québécois, paraissent loin d'être épuisées. Ainsi, une organisation comme les Cercles des jeunes naturalistes (CJN) a été fréquemment citée pour illustrer la force, la profondeur et la vitalité de l'effort scientifique au Québec, surtout pendant les années 1930 et 1940. Des chiffres tout à fait étonnants sur la multiplication des cercles à partir de 1931 ont été souvent avancés dans l'historiographie (532 cercles pour 1934'1 882 CJN et 25000 membres pour $1940^{2}$, «1 389 cercles, dont 815 vraiment actifs» en $1954^{3}$ ) et leur ont valu une image de puissant mouvement de masse ${ }^{4}$, agissant, à l'époque, comme une véritable base sociale pour un mouvement scientifique canadien-français (au sens du «scientistic movement» de J. Ben-David ${ }^{5}$ ) et comme caution pour certains scientifiques, notamment pour le frère Marie-Victorin alors en conflit avec l'Institut scientifique franco-canadien ${ }^{6}$.

Or, il appert que les CJN qui, aujourd' hui encore, poursuivent leur action parmi les clubs scientifiques au Québec, étaient mal connus: en fait, notre article, qui présente quelques-uns des résultats d'une recherche plus étendue, vise à préciser certains points comme l'importance, la nature et la portée du mouvement ${ }^{7}$. Le traitement informatique d'une partie du fonds d'archives des CJN permet ici non

1. Jean-Claude Guédon, «Du bon usage de la vulgarisation, le cas de Marie-Victorin», Questions de Culture, 1 (1981): 101.

2. Luc Chartrand, Raymond Duchesne et Yves Gingras, Histoire des sciences au Québec (Montréal, Boréal, 1987), 264.

3. Luc Chartrand, «Ces chers ancêtres, les croisés des sciences naturelles», Québec science (mai 1981): 54.

4. L'expression est fréquente. Voir entre autres Luc Chartrand, Raymond Duchesne et Yves Gingras, op. cit., 263; P. Carle et J.-C. Guédon, «Vulgarisation et développement des sciences et des techniques - le cas du Québec (1850-1950)», dans Daniel Jacobi et Bernard Schiele, dir., Vulgariser la science, le procès de l'ignorance (Seyssel, Éditions du Champ Vallon, 1988), 207.

5. Joseph Ben-David, The Scientist's Role in Society (Englewood Cliffs, NJ, PrenticeHall, Inc., 1971), 207 p.

6. Cette thèse est notamment défendue par Jean-Claude Guédon, loc. cit., 81-111. Voir aussi Marcel Fournier et Louis Maheu, «Nationalisme et nationalisation du champ scientifique québécois», Sociologie et Sociétés, 7,2 (1975): 89-113.

7. Voir Pierrick Malissard, Le mouvement scientifique au Québec et les Cercles des jeunes naturalistes, 1931-1971, mémoire de maîtrise, Université du Québec à Montréal, 1993, 243 p. 
seulement d'évaluer l'ampleur du mouvement année après année, mais aussi de clarifier l'image des milieux où se sont développés les cercles et de mettre en lumière certaines caractéristiques de la clientèle touchée par les CJN, notamment sa répartition géographique.

\section{ORIGINE DU MOUVEMENT}

Créés en février 1931 sous l'égide de la Société canadienne d'histoire naturelle (SCHN), les CJN se voulaient une méthode novatrice en matière d'enseignement scientifique. Axés sur les travaux pratiques, les excursions de groupe et les sciences d'observation (botanique, entomologie, zoologie, ornithologie, en particulier), les cercles visaient à initier leurs membres à l'étude de la nature, entre autres par la constitution d'herbiers ou de collections entomologiques. L'approche n'était pas neuve: depuis le début du siècle, certaines maisons d'enseignement religieuses (comme le juvénat de Saint-Bruno des Frères de Saint-Gabriel) complétaient la formation de leurs élèves par ce moyen. Dès le milieu des années 1920, un enseignant de Ville Saint-Laurent, le frère Adrien, de la Congrégation de Sainte-Croix, avait rodé, dans son école, une formule dérivée des clubs Audubon, qui allait servir de modèle pour les CJN. Membre de la SCHN, le frère Adrien y côtoyait des universitaires, Jacques Rousseau et le frère Marie-Victorin en particulier, qui, reprenant l'idée du club scientifique, allaient lui donner, grâce à leur «capital scientifique» et à leurs appuis dans la presse (au Devoir surtout), une impulsion décisive au niveau provincial. En fait, l'approche du frère de Sainte-Croix rejoignait parfaitement les critiques et les propositions de Marie-Victorin - et de Jacques Rousseau - contre un enseignement «trop livresque» dans les années 1920. Dès lors, et pendant au moins une dizaine d'années, les cercles allaient devenir un élément important dans les stratégies d'institutionalisation des chefs de file de la SCHN et de l'Association canadienne-française pour l'avancement des sciences (ACFAS) ${ }^{8}$. Le frère Marie-Victorin ne manqua jamais de rappeler l'ampleur du mouvement des CJN dans ses plaidoyers pour la création du Jardin botanique de Montréal, par exemple. De plus, les grandes expositions des CJN, qui semblent avoir attiré des foules importantes, furent organisées de façon à coïncider avec les premiers congrès de l'ACFAS, la conjonction des événements multipliant ainsi

8. L'ACFAS consacrera d'ailleurs, pendant deux ou trois ans, la moitié de son budget de mille dollars au CJN. Voir le discours de Jacques Rousseau, «Un quart de siècle à la Société canadienne d'histoire naturelle», 22 février 1949, 11. Archives des CJN (ACJN), Service des archives de l'UQAM, 16P12a/29. 
les effets de publicité et pour les cercles et pour la communauté scientifique.

Si plusieurs universitaires, Marie-Victorin en tête, jouèrent un rôle crucial dans le décollage du mouvement, ils intervinrent assez peu dans le détail du fonctionnement des cercles. La SCHN laissa toujours aux CJN une liberté à peu près totale quant à l'organisation de leurs activités, ne s'attribuant «que le droit de les aider de toutes [ses] forces ${ }^{9} »$. Cette aide, sous forme de tracts et de publications gratuites (du moins dans les premiers temps), fut cependant soumise à certaines conditions: d'abord, demander leur affiliation auprès de la SCHN, et ensuite faire parvenir, tous les ans, un compte rendu de leur fonctionnement au secrétariat général des CJN à Montréal.

Cette dernière exigence paraît avoir été relativement bien suivie pendant plus de quarante ans et on peut retrouver aujourd'hui, regroupés dans chaque dossier de cercle, ces rapports qui constituent des témoignages tangibles de la vitalité passée des CJN. Les archives comptent ainsi 1959 dossiers, renfermant la correspondance, les pièces administratives et les rapports d'activités de chaque cercle pour les années 1931 à 1978, qui ont été systématiquement dépouillés pour cette étude. Ces fichiers de cercles représentent probablement la seule possibilité d'évaluer l'ampleur du mouvement. Le secrétariat général, qui n'a jamais eu d'autres moyens plus fiables pour estimer le nombre des cercles, a cependant rarement pu, surtout par manque de personnel, en garder le compte exact. De plus, la direction des CJN a longtemps laissé la définition du cercle dit «actif» dans le vague, distinguant, à l'occasion, les cercles «ralentis» ou «inactifs» des cercles «morts»; dans le cadre de la présente étude, un cercle est réputé «actif» s'il existe un rapport pour l'année courante. Dans cette perspective, un des intérêts de notre approche sera de confronter nos résultats avec les chiffres qui figurent dans les publications et les nombreux articles sur les cercles dans la presse de l'époque, chiffres généralement fournis par l'exécutif des CJN et repris, plus tard, par l'historiographie. De plus, la grande homogénéité des sources permettent, malgré quelques faiblesses, de suivre le mouvement de façon continue sur plus de 40 ans.

La présente étude a donc été développée principalement autour de cinq grandes questions:

1) le nombre des cercles et de leurs membres de 1931 à 1971;

9. Marie-Victorin, «Les Cercles des jeunes naturalistes, il faut fonder un CJN», Tract 1, Bibliothèque des jeunes naturalistes, 30 janvier 1932. 
2) la répartition des cercles entre les secteurs laïque et religieux, d'une part, et, selon les congrégations enseignantes, d'autre part;

3) la répartition des membres selon le sexe;

4) la moyenne d'âge des membres de 1953 à 1971, la répartition des cercles entre les différents niveaux scolaires et entre les différents types d'établissements;

5) la distribution géographique des cercles.

\section{LE NOMBRE DE CERCLES ET DE MEMBRES DE 1931 À 1971}

De prime abord, même si l'exécutif des CJN l'a largement surestimée, la croissance des cercles fut exceptionnelle et ce, dès les premières années (figure 1): touchant pratiquement exclusivement des établissements scolaires dirigés ${ }^{10}$ par des congrégations religieuses, cette progression apparaît résolument forte jusqu'en 1939, malgré un léger tassement vers 1934-1935. Il est vraisemblable qu'une telle augmentation ait surpris les initiateurs du mouvement eux-mêmes, suscitant l'idée d'une vague de fond souvent évoquée dans la presse.

Le déclin des années de guerre et la stagnation de l'immédiat après-guerre ne sont peut-être pas surprenants: les sciences naturelles ne constituant pas une préoccupation majeure pendant ces années troublées. L'on remarque cependant que cette période correspond à un effort important de la communauté scientifique au Québec qui, grâce à Radio-Collège, tenta de diffuser la science auprès d'un large public. De toute évidence, les émissions La cité des plantes et Le monde animal n'ont pas empêché une chute sensible du nombre des cercles.

Plus étonnant est le brusque sursaut de 1948: une augmentation de $52 \%$ en une année ( $+66 \%$ pour les membres) constitue un bond considérable. Le décompte des cercles par congrégation, qui sera analysé plus loin, permettra de cerner où se produit cette poussée attribuable sans doute à une réforme des programmes scolaires visant, entre autres, à améliorer l'enseignement des sciences. L'année 1948 marqua, en effet, le début d'une forte reprise qui ne prit fin qu'avec l'effondrement du mouvement pendant la Révolution tranquille.

Si l'examen du nombre des fondations de CJN jusqu'aux années 1960 montre des variations de forte amplitude, le décompte des disparitions de 1931 à 1971 suggère un taux de renouvellement des cercles qui reste toujours relativement élevé. Le problème paraît d'ail-

10. Ou bien parfois, dans lesquels l'enseignement était assuré par des religieux. 
FIGURE 1

Nombre de cercles actifs de 1931 à 1971

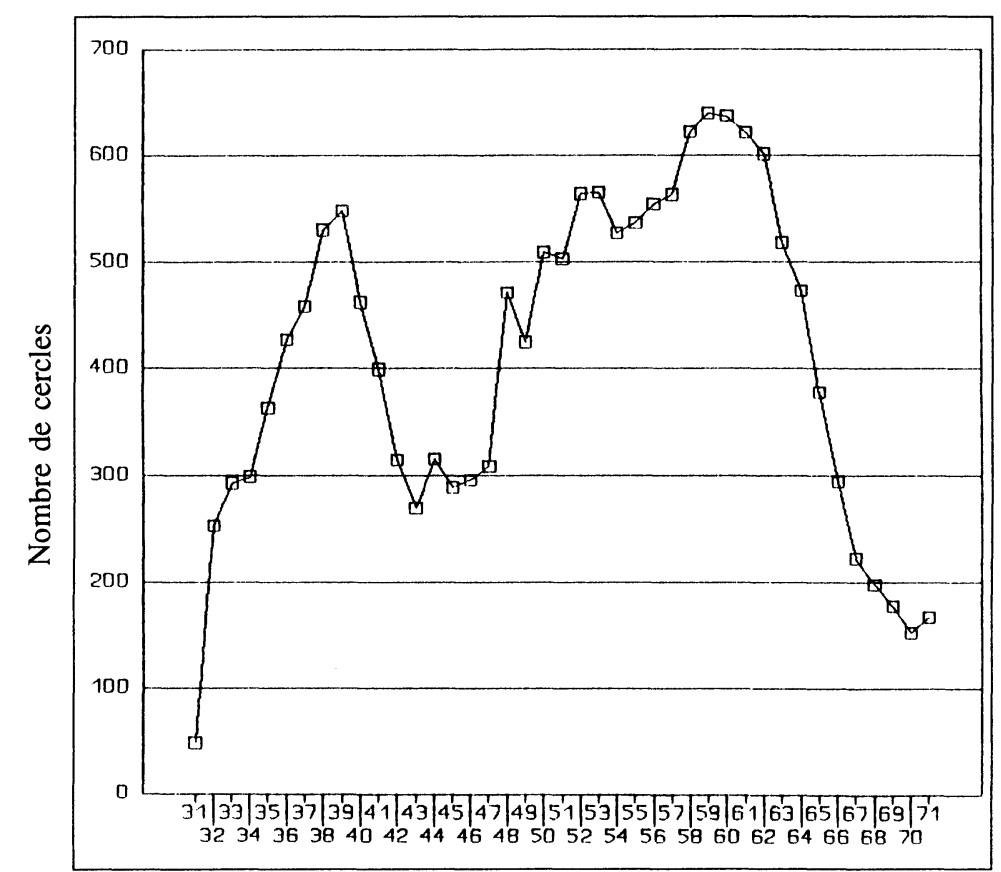

Années

Source: ACJN, 16P5b/1-1959.

leurs suffisamment endémique pour que la direction des CJN adresse assez régulièrement des mises en garde contre les «feux de paille» et lance des appels à la persévérance ${ }^{11}$.

Les années 1950 ont vu une forte hausse de la moyenne du nombre de membres par cercle alors que l'accroissement du nombre de cercles, très prononcé jusqu'en 1952, semble plafonner par la suite. Très vraisemblablement, le facteur démographique est intervenu largement dans la croissance du mouvement: la population scolaire du Québec s'est presque multipliée par trois entre 1940 et 1960, sans que $16 \mathrm{P} 5 \mathrm{a} / 3$.

11. Voir, entre autres, la circulaire aux directeurs de CJN, 23 mai 1947, ACJN, 
les CJN n'améliorent sensiblement leur position relative ${ }^{12}$ dans des établissements de plus en plus importants en termes d'effectifs. Seul augmentait alors le nombre de membres par cercle: 16748 membres pour 530 cercles en 1938, 24671 pour 527 CJN en 1954.

Le zénith du mouvement en 1961 précéda presque immédiatement la chute brutale des CJN qui se poursuivit tout au long des années 1960 et 1970. La laïcisation du système scolaire québécois et la refonte des programmes, en particulier de l'enseignement des sciences, ont, semble-t-il, porté un coup très dur aux cercles. Après 1970 et jusqu'à aujourd'hui, le nombre de CJN se stabilisera autour d'une centaine.

Ces résultats, qui attestent la grande vitalité des cercles pendant les années 1930, infirment cependant les chiffres annoncés dans la presse de l'époque ${ }^{13}$ et repris, par la suite, dans l'historiographie: en fait, ce n'est que dans les années 1960 que le nombre de jeunes naturalistes franchira la barre des 30000 membres, chiffre fréquemment évoqué dans Le Devoir dès $1940^{14}$. La direction des CJN privilégia pendant très longtemps un décompte cumulatif des fondations de cercles, sans tenir compte des disparitions, semble-t-il, ce qui eut pour effet de gonfler les chiffres (848 CJN en 1939, 925 en 1943, 1391 en 1955 contre 548, 269, 537 pour les mêmes dates dans notre étude, laquelle, rappelons-le, ne compte que les cercles ayant soumis un rapport annuel). Après la guerre, la nouvelle direction essaya de rectifier les statistiques probablement pour se donner une vision plus juste du mouvement. Des différences subsistent malgré tout avec notre compilation; une certaine souplesse, de la part de la direction des CJN, dans la définition des cercles «actifs» l'explique sans doute ${ }^{15}$.

Sans être responsables de la distorsion, les chefs de file de la SCHN et de l'ACFAS l'ont manifestement utilisée. Très peu nombreux et souvent débordé, le personnel du secrétariat des CJN n'a pratiquement jamais pu tenir la liste des cercles à jour. Il demeure qu'il était naturellement de l'intérêt de Marie-Victorin et du groupe de

12. Tout au long de la période, les CJN ne seront jamais présents dans plus de 5 à $6 \%$ des écoles du Québec.

13. Pratiquement toujours dans des articles signés par des responsables de la SCHN et de l'ACFAS, Marie-Victorin, Jacques Rousseau, Henri Prat, ou par des journalistes reprenant leurs propos.

14. Voir, par exemple, Le Devoir, 16 janvier 1940; un article signé A. Ayotte annonce 825 cercles et 100000 membres en 1939, «500 000 personnes touchées», Le Devoir, 7 juin 1939.

15. Les conventions alors adoptées par le secrétariat des CJN sont décrites dans: Sœur Marie-Jean-Eudes, ssa, Les Cercles des jeunes naturalistes. Pages d'histoire (Lachine, Éditions Sainte-Anne, 1981), 127. 
l'Institut botanique d'entretenir une estimation optimiste du nombre de cercles. Engagés dans de délicates négociations avec le gouvernement provincial pour faire avancer leurs projets, le Jardin botanique entre autres, pour assurer un financement stable à l'ACFAS et se démarquer de l'IFSC, ces universitaires se sont efforcés de créer, en évoquant ces grands nombres, un effet mobilisateur. En outre, pour d'autres raisons touchant la réputation de leur enseignement des sciences, il était également de l'intérêt des communautés enseignantes de cultiver l'impression d'un renouveau général où elles jouaient un rôle moteur. Comme l'indique, par exemple, ce commentaire d'un journaliste du Devoir à l'occasion de l'exposition des CJN de 1933, les réussites du mouvement rejaillissaient aussi sur les religieux: «Le succès souligne une fois de plus aussi la puissance du merveilleux instrument que peuvent être pour toute œuvre de progrès nos congrégations enseignantes ${ }^{16} . »$

Dans ce contexte, où personne ne pouvait ni ne voulait clarifier la situation, les CJN allaient baigner dans un sfumato qui, voilant l'ampleur et la véritable nature du mouvement, se perpétua jusque dans l'historiographie récente.

\section{LA RÉPARTITION DES CERCLES ENTRE LES SECTEURS LAÏQUE ET RELIGIEUX}

La prédominance des communautés enseignantes dans le mouvement apparaît clairement (figure 2) et laisse voir une relative indifférence des milieux laïques à l'égard des CJN.

\section{Le secteur laïque}

Mise à part une modeste poussée entre 1936 et 1942, les cercles laïques, qui furent, sauf vers la fin des années 1960, d'abord des cercles d'écoles rurales, plafonnèrent, sur une base annuelle, autour d'une quarantaine tout au long de la période. En fait, les enseignants laïques, des enseignantes dans la plupart des cas, paraissent avoir été très réticents face aux CJN: déjà sous-payés, peu d'entre eux acceptèrent d'encadrer bénévolement des jeunes dans ce mouvement. Il y eut des exceptions cependant, et il faut souligner ici les efforts d'institutrices comme Blanche Paradis au Lac-Saint-Jean et RoseEmma Heppell dans la vallée de la Matapédia qui, dans les années 1940 surtout, réussirent, en plus d'animer leur propre cercle, à susciter, chacune dans leur région, la création de plusieurs dizaines de

16. Article d'Omer Héroux, Le Devoir, 16 décembre 1933. 
FIGURE 2

Répartition des cercles entre les secteurs clérical et laïque de 1931 à 1971

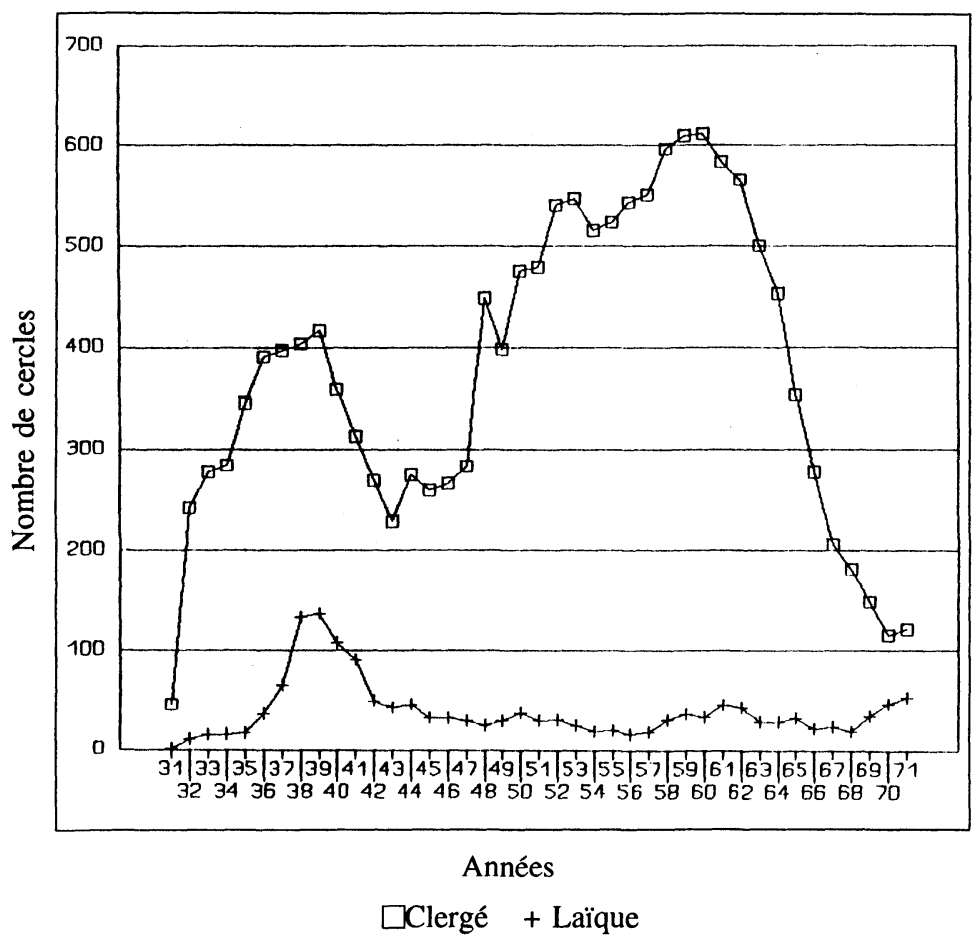

Source: ACJN, 16P5b/1-1959.

CJN. Le rôle de certains inspecteurs d'écoles fut probablement déterminant; ainsi, par exemple, à la même époque, en Gaspésie, l'inspecteur régional, Paul Hubert, encourageait les inspecteurs de district à stimuler les fondations de CJN auprès des enseignants ${ }^{17}$.

De plus, les écoles normales ont pu avoir une influence: découvrant les CJN pendant leurs années de formation, les jeunes enseignants furent parfois tentés de s'engager dans le mouvement dès leurs premières affectations ${ }^{18}$. Dans ce sens, le dynamisme des cercles

17. Voir Archives des Sœurs de Notre-Dame du Saint-Rosaire, Rimouski (ARSR), 820.281.C3.70a.

18. Les demandes d'affiliation des cercles sont souvent révélatrices; voir, entre autres, ACJN, 16P5b/978, /992, /1608, /1720. 
laïques témoigne aussi des efforts des communautés qui maintenaient ces écoles normales.

Les mutations dans la société québécoise des années 1960 ayant entraîné un déclin rapide du rôle des congrégations enseignantes, les cercles laïques, tout en conservant le même niveau, devinrent majoritaires dans les années 1970. Dès lors, la plupart des cercles vont se développer, soutenus par des bénévoles laïques (et par quelques religieux), à l'extérieur de tout cadre scolaire, à l'instar de la plupart des autres clubs scientifiques pour la jeunesse.

\section{Le secteur religieux}

En fait, c'est la participation des religieux qui va donner au mouvement toute son ampleur. D'après notre compilation, des religieux d'environ 50 communautés différentes se sont engagés auprès des cercles. Nous avons retenu, dans la présente étude, les 15 congrégations les plus représentées, les autres étant rassemblées dans un groupe «divers religieux» indifférencié; une catégorie «prêtres» a éga-

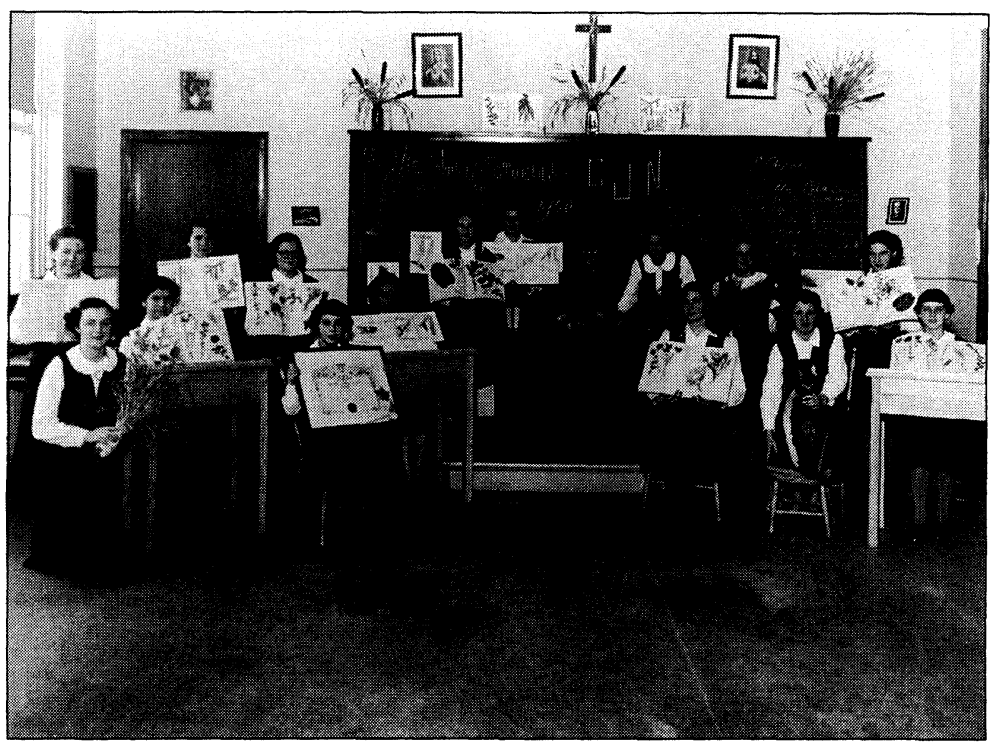

Saint-Joseph de Hull, 1950. Services des archives de l'UQAM, Fonds CJN. 
lement été constituée qui regroupe surtout des professeurs de séminaire ${ }^{19}$.

Cette recherche montre surtout l'importance des congrégations féminines dans le mouvement: tout au long de la période étudiée, le directeur de cercle fut le plus souvent une religieuse. Il est à noter toutefois que, si l'on s'en tient au nombre de cercles, le rapport entre religieux et religieuses dans les CJN (presque 77\% de sœurs) n'est pas très éloigné de ce même rapport dans le système éducatif provincial (environ 72\% de sœurs en 1940-1941, idem en 1949-1950). Cependant, quand on considère les effectifs des cercles sous la responsabilité des religieuses, le poids des communautés féminines s'avère écrasant.

Cette répartition des CJN entre les congrégations a eu, de surcroît, des répercussions sur la clientèle des cercles: les Dames de la Congrégation de Notre-Dame (CND) ou les Sœurs de Sainte-Anne (SSA) ne recrutaient, ni dans les mêmes milieux, ni pour les mêmes niveaux scolaires, ni dans les mêmes régions que les Sœurs de Notre-Dame du Saint-Rosaire (RSR), par exemple ${ }^{20}$. Examinée tout au long de la période étudiée, cette répartition des cercles entre les communautés laisse voir de très fortes variations: chaque congrégation semble avoir connu de brusques poussées qui vinrent constamment modifier sa position relative (figures 3 et 4). En fait, tout se passe comme si les cercles apparaissaient subitement dans la plupart des établissements d'une même congrégation. Ainsi la place des CND en 1936 (figure 3) a-t-elle fait suite à trois années de forte croissance au cours desquelles 93 CJN furent fondés dans cette communauté. De même, le sursaut des cercles en 1948 s'explique par la participation soudaine de deux congrégations, les Religieuses de Sainte-Croix (RSC) et les Sœurs de Saint-Joseph de Saint-Hyacinthe (SJSH), à qui l'on doit la mise en place de près de $100 \mathrm{CJN}$ (figure 4).

Au-delà du nombre de créations de CJN, les communautés se distinguent aussi par la grande persistance de leurs cercles: chez les

19. Il s'agit de la Congrégation de Notre-Dame (CND), la Congrégation de Sainte-Croix (CSC), des Clercs de Saint-Viateur (CSV), des Filles de la Charité du Saint-Cœur de Jésus (FCSCJ), des Frères des Écoles chrétiennes (FEC), des Frères Maristes (FM), des Sœurs de la Présentation de Marie (PDM), des Religieuses de Jésus et de Marie (RJM), des Religieuses de Sainte-Croix (RSC), des Religieuses du Saint-Rosaire (RSR), des Frères du Sacré-Cœur (SC), des Frères de Saint-Gabriel (SG), des Sœurs de Saint-Joseph de Saint-Hyacinthe (SJSH), des Sœurs des Saints Noms de Jésus et de Marie (SNJM), des Sœurs de Sainte-Anne (SSA). Le groupe «divers religieux» est représenté sur les figures 3 et 4 par l'abréviation REL; PTRE désignant le groupe des prêtres.

20. Micheline Dumont et Nadia Fahmy-Eid, dir., Les couventines, l'éducation des filles au Québec dans les congrégations religieuses enseignantes, 1840-1960 (Montréal, Boréal, 1986), 193. 
FIGURES 3 ET 4

Répartition des cercles entre les différentes congrégations enseignantes et le secteur laïque en 1936 et 1951

1936

Nombre de cercles

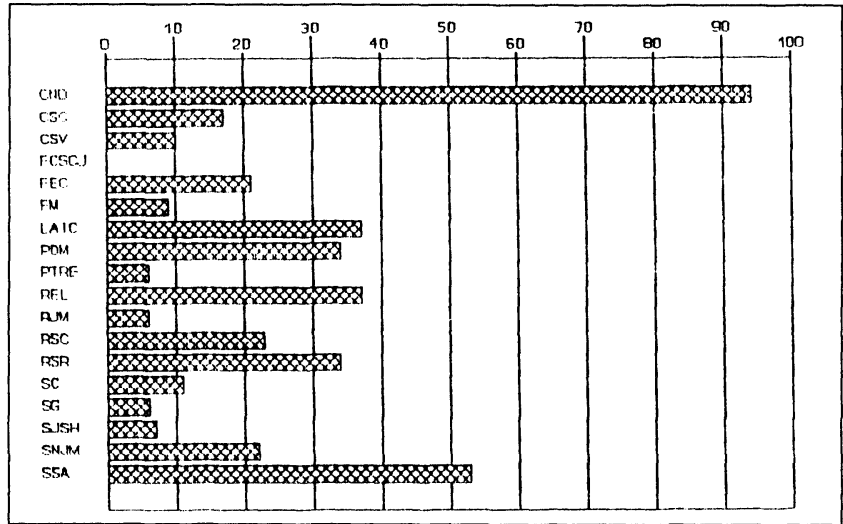

1951

Nombre de cercles

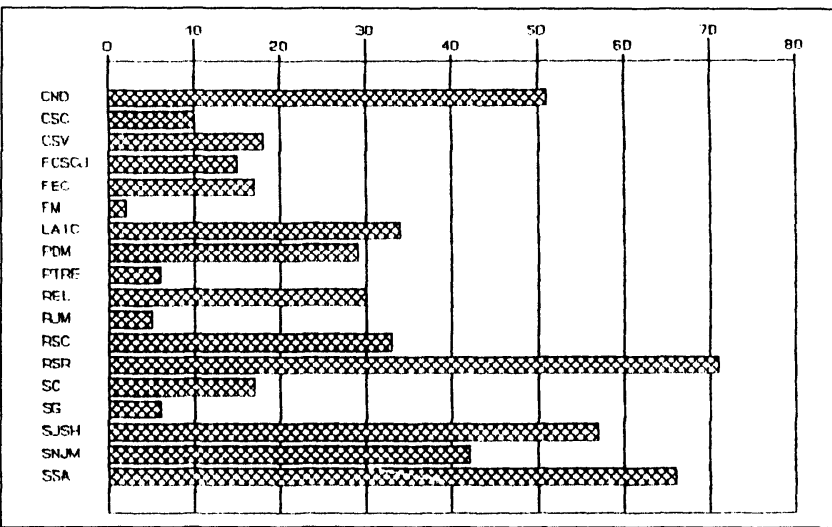

Voir note 19 pour les abréviations.

Source: ACJN, 16P5b/1-1959. 
Clercs de Saint-Viateur (CSV), la durée de vie moyenne d'un CJN est de 10,6 ans, 15,3 ans chez les Frères des Écoles chrétiennes (FEC), 16 ans dans la Congrégation de Notre-Dame, 26,5 ans chez les Sœurs du Saint-Rosaire et même 27,8 ans chez les Sœurs de Sainte-Anne; en comparaison, les cercles laïques avec 6,4 années se révèlent très fragiles. De plus, il est très rare qu'un cercle initialement organisé par des religieux soit repris par des laïcs; la laïcisation du système scolaire dans les années 1960 en fera donc disparaître un grand nombre.

De toute évidence, ce fut donc, le plus souvent, au plus haut niveau dans la congrégation qu'était prise - ou du moins autorisée la décision de fonder des cercles: les CJN apparaissent souvent soudainement et massivement dans la plupart des écoles sous la responsabilité d'une communauté. De plus, dans plusieurs communautés féminines, une responsable des cercles, qui servit d'intermédiaire avec le secrétariat des CJN, fut d'ailleurs désignée.

Les causes de l'adhésion des congrégations au mouvement furent extrêmement variées. Elles dépendaient d'abord certainement à la fois de la position des communautés dans le champ du système éducatif au Québec et de leur position dans le champ religieux: a priori, l'initiative d'un frère de Sainte-Croix, le frère Adrien, même appuyée par le frère Marie-Victorin - qui restait un frère des Écoles chrétiennes -, avait peu de chances de séduire les prêtres enseignants dans les collèges classiques, par exemple. De fait, les CJN ne furent jamais très nombreux dans ces institutions (au plus fort de leur implantation en 1960-1961, ils étaient présents dans 19 des 37 collèges classiques de garçons). Ce constat effectué, l'on n'a probablement pas épuisé la complexité des motivations des religieux pour fonder des cercles puisque des membres d'ordres prestigieux comme la congrégation de Saint-Sulpice ou la Société de Jésus s'impliquèrent parfois dans le mouvement: c'est d'ailleurs un jésuite, le père Sénécal, qui assura, avec deux autres religieux, la direction des CJN de 1955 à 1976.

En fait, dans les années 1920 et 1930, l'attitude de l'Église face à l'enseignement des sciences demeura extrêmement complexe et ce, d'autant plus qu'elle impliquait une multitude de communautés enseignantes qui, parfois, ne relevaient même pas du droit diocésain (ou pouvaient se prévaloir de privilèges pontificaux) et qui répondaient aux directives ou aux souhaits de l'épiscopat avec plus ou moins d'empressement ou d'efficacité. Chaque congrégation disposait, en effet, d'une relative autonomie, très limitée mais réelle, qui s'exercait par l'intermédiaire de sa préfecture générale des études ${ }^{21}$ dont le rôle

21. Les appellations et la forme pouvant toutefois grandement varier, il sera fait référence ici aux communautés les plus importantes numériquement. 
était de diriger, d'orienter et d'uniformiser, au sein de la communauté, toute l'action pédagogique des religieux enseignants.

De plus, chaque congrégation tendait à entretenir, dans ses méthodes, une certaine spécificité dont le fondement procédait sans doute d'abord d'une volonté, de la part des religieux enseignants, de s'affirmer face au secteur public et aux exigences gouvernementales (comme le suggère la corrélation entre la réforme de 1948 et la soudaine poussée des CJN) et, en second lieu, d'un climat d'émulation entre les communautés qui se devaient de faire bonne figure «aux yeux des parents ${ }^{22} \gg$. L'émulation pouvait parfois faire place à une certaine concurrence, comme dans le cas des Frères des Écoles chrétiennes qui luttèrent de nombreuses années pour faire reconnaître à leurs maisons d'enseignement les droits et privilèges des collèges classiques. Les efforts des «Ignorantins» portèrent, entre autres, sur le Mont-Saint-Louis qui était réputé, dans les années 1920 et 1930, pour la qualité de son enseignement des sciences ${ }^{23}$.

Initiative de la communauté scientifique, les cercles firent l'objet d'un démarchage systématique auprès des préfectures générales des études des congrégations: appuyé par des articles des responsables de la SCHN et de l'ACFAS dans la presse, principalement dans Le Devoir, le premier directeur des CJN, le frère Adrien, se livra littéralement à du porte-à-porte pendant une bonne partie des années 1930 . Présentés comme une méthode moderne d'enseignement, les CJN allaient alors constituer dans les premiers temps, pour les communautés qui en ressentaient le plus le besoin, un moyen d'améliorer, à peu de frais, l'image - et la qualité - de leur enseignement scientifique grâce à la caution d'universitaires connus.

La coopération entre les scientifiques et les religieux fluctua cependant dans le temps. Très probablement envisagée au début par les chefs de file de la SCHN comme une expérience transitoire, destinée à être complétée par une réforme en profondeur de l'enseignement des sciences, les cercles s'installèrent dans une permanence ambiguë. Il est clair que pour Marie-Victorin, par exemple, les CJN ne devaient être qu'un premier pas; son discours présidentiel au congrès de l'ACFAS de 1938 donne le ton:

J'ai entendu d'honnêtes idéalistes exprimer l'idée que le vide scientifique [...] sera comblé par des mouvements tels que le scoutisme, les cercles des jeunes naturalistes [...].

22. Selon le mot de Sœur Sainte-Théophanie, cnd, cité dans Micheline Dumont et Nadia Fahmy-Eid, dir., op. cit., 116.

23. Claude Marcil et André Lemelin, "Une histoire de l'éducation au Québec», Éducation Québec, 11,1 (septembre 1980): 43. 
[...] Mais quand les enfants seront couchés, nous les vieux, regardons-nous franchement.

Nous ne sortirons pas de l'impasse par des expédients, mais par un mouvement profond [de réforme de l'éducation] [.... ${ }^{24}$

De plus, selon le président de l'ACFAS, l'enjeu principal se situait au niveau de l'enseignement universitaire: «Il en est de l'enseignement comme de la pluie du ciel: l'un et l'autre tombent de haut en bas, et la bonne ordonnance de l'enseignement supérieur retentit sur l'enseignement secondaire et [...] primaire $^{25}$.»

Cette perspective montre assez l'écart entre le projet envisagé par Marie-Victorin et les intentions des religieux. De fait, pour les responsables des communautés enseignantes, dont les décisions n'étaient pas orientées au premier chef par une vision globale du problème des sciences au Québec et qui, de toutes manières, étaient peu intéressés à bouleverser le système éducatif, les CJN constituaient une fin en soi: les cercles venaient compléter, sans les déranger, les plans d'études des religieux. La réforme de 1948 allait même, dans la pratique, entériner cette conception des $\mathrm{CJN}$ : mis en place par le département de l'Instruction publique, le nouveau programme n'apportait pas de grands changements en matière d'enseignement des sciences. Cependant, les directives pédagogiques générales accompagnant la réforme insistaient, pour intéresser les enfants aux sciences, sur la nécessité de créer des cercles de jeunes naturalistes et conseillaient «d'organiser des promenades où l'on pourra concrétiser les leçons de choses données en classe ${ }^{26} »$. Applaudi par certains enseignants religieux du primaire et du secondaire ${ }^{27}$, ce nouveau programme fut très probablement une des causes du brusque sursaut du nombre de cercles observé après 1948.

Dans ce contexte, dès les années 1950 , l'appui des scientifiques aux CJN allait se tarir. De fait, il se produisit un certain renouvellement des effectifs de la SCHN: des personnalités disparurent ${ }^{28}$ ou se

24. Marie-Victorin, La science et notre vie nationale, discours présidentiel, congrès de l'ACFAS, 1938, extrait, avec changement de pagination, des Annales de l'ACFAS, 5 (1939): 136155. (Montréal, [s.n.], 1939), 17.

25. Marie-Victorin, «Les sciences naturelles dans l'enseignement supérieur», Le Devoir, 6 et 7 octobre 1930.

26. Conseil de l'Instruction publique, Programme d'études des écoles primaires élémentaires, approuvé par le Comité catholique du Conseil de l'Instruction publique le sept mai dix-neuf cent quarante-huit (Québec, Imprimerie du Gouvernement), 416.

27. Voir, entre autres, Sœur Sainte-Marie-Médiatrice, Un nouvel intérêt pédagogique: les cercles des jeunes naturalistes (Montréal, Éditions de la nature, 1949), 84 p. ACJN, 16P12a/28.

28. Léo Pariseau s'éteint en janvier et Marie-Victorin décède d'un accident de voiture en juillet 1944 . 
retirèrent, comme Jacques Rousseau qui quitta la direction de la Société en 1949, et une nouvelle génération, pas nécessairement au sens biologique, vint prendre la relève. De plus, l'Institut botanique, le Jardin botanique de Montréal et le Jardin zoologique de Québec étaient là pour témoigner du chemin parcouru, de la reconnaissance sociale obtenue par la communauté scientifique dans le domaine des sciences naturelles. Le militantisme des premières années de la Faculté des sciences avait décliné et les universitaires, avec certes des exceptions, furent de moins en moins nombreux à s'engager directement dans des cercles. Pour faire un parallèle avec l'analyse de Luc Boltanski, cette réduction de leur visibilité sociale fut peut-être un des signes de l'autonomisation de leur espace scientifique ${ }^{29}$. De toute évidence, les scientifiques n'avaient plus véritablement d'intérêt à s'impliquer dans les CJN à partir des années 1950 . On peut même se poser la question de savoir si les cercles, du fait de leurs liens avec les religieux, ne devinrent pas, pour les scientifiques, une activité exotérique qui ne cadrait plus avec l'image qu'ils cherchaient à donner d'eux-mêmes ${ }^{30}$. Dès lors, le sort des CJN se trouvera largement lié à celui du clergé dans le système éducatif au Québec; à partir du déclin de l'influence des religieux dans les écoles, les cercles se rétracteront autour du noyau des animateurs laïques. Comme le souligne le père Dollard Sénécal, de la Compagnie de Jésus, qui fut à la direction des CJN de 1955 à 1976: «On peut dire ce qu'on voudra des frères et des sœurs, mais ils avaient du temps 'en masse' pour s'occuper des enfants, même en dehors des heures régulières de $\operatorname{cours}^{31}$.»

De fait, l'effondrement des cercles fut provoqué probablement davantage par une pénurie d'animateurs que de membres. Par ailleurs, on peut penser que le mouvement a peut-être bénéficié de la vogue écologiste des années 1970: la protection de la nature devint alors, en effet, un thème récurrent pour les CJN.

29. Selon Boltanski, «plus un champ est autonome, moins les détenteurs du 'pouvoir local' disposent (...) d'une surface sociale et d'un 'pouvoir général' étendus». Même si les CJN sont une «position de pouvoir» assez marginale, ils illustrent aussi la dispersion des efforts à laquelle sont contraints les scientifiques d'un domaine peu autonomisé. Voir Luc Boltanski, «L'espace positionnel. Multiplicité des positions institutionnelles et habitus de classe», Revue française de sociologie, 14 (1973): 12.

30. Voir, par exemple, Pierre Dansereau, «Lettre à un séminariste sur l'aliénation des intellectuels», Cité libre, 11,32 (décembre 1960): 14-17, où sont exprimées les tensions entre les scientifiques et l'Église.

31. Cité dans Perspective, 24 mai 1975, 18. ACJN, 16P14/15. 


\section{LA RÉPARTITION DES MEMBRES DE CJN SELON LE SEXE}

Le dynamisme des religieuses et surtout la présence massive des filles dans le mouvement sont probablement une des surprises de cette recherche (figure 5). De 1931 à 1971, les cercles féminins ont constamment dominé le mouvement et dans des proportions tout à fait écrasantes certaines années (autour de 90\% en 1956). En fait, la croissance des CJN, pendant les années 1950, a été provoquée en grande partie par une poussée fulgurante des filles dont les cercles, par rapport aux garçons, furent à la fois plus nombreux, plus stables et plus importants en termes d'effectifs. Cette disproportion a peut-être des causes complexes, mais elle est très certainement liée à l'attitude des religieuses face aux CJN. Cette forte représentation des congrégations féminines dans un mouvement prônant les sciences illustre peut-être un aspect méconnu du rôle des religieuses ${ }^{32}$ : au-delà des

FIGURE 5

Répartition des membres selon le sexe de 1931 à 1971

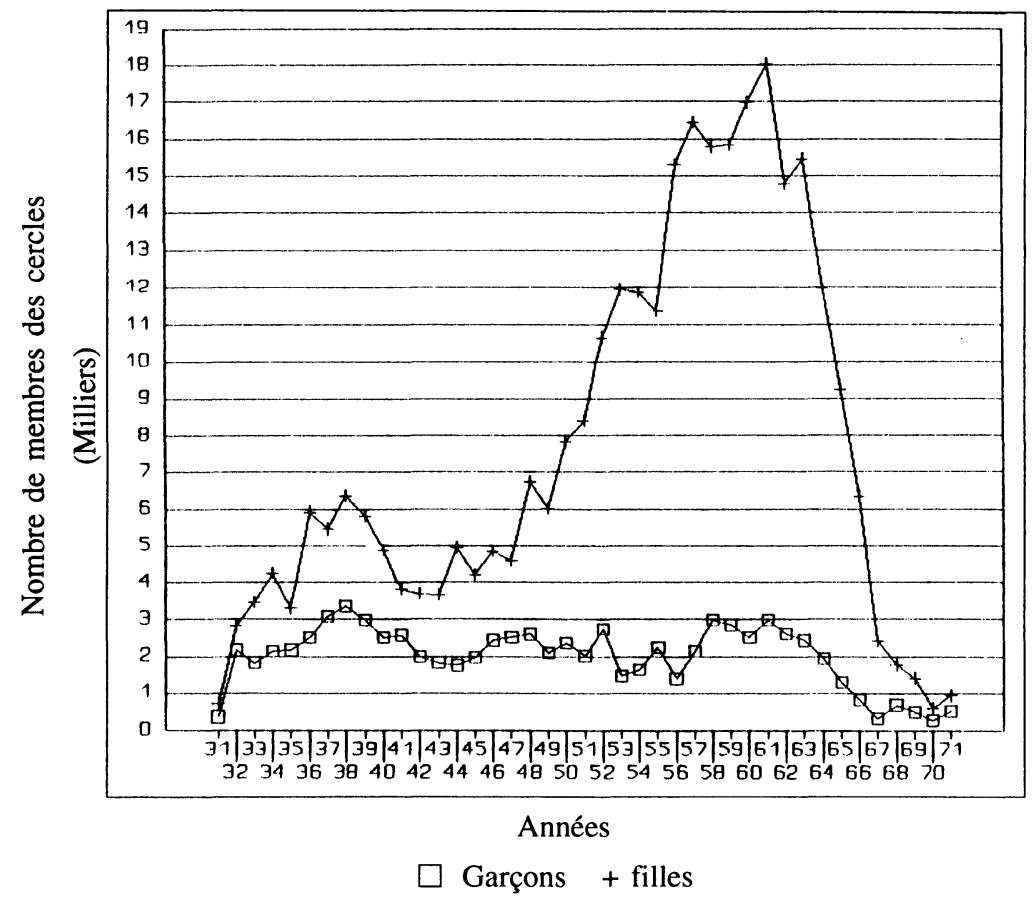

Source: ACJN, 16P5b/1-1959.

32. Danielle Juteau-Lee, «Les religieuses du Québec: leur influence sur la vie professionnelle des femmes, 1908-1954», Atlantis, 5,2 (printemps 1980): 22-33. 
stéréotypes, les communautés féminines ont peut-être eu effectivement une position relativement avant-gardiste dans l'enseignement pour jeunes filles. Au reste, les filles se signalèrent systématiquement à tous les concours des CJN. En fait, les résultats du concours de botanique du Devoir en $1930^{33}$, qui amorça le mouvement, suggèrent même que les filles bénéficiaient, bien avant les CJN, d'une initiation aux «petites sciences» vraisemblablement supérieure à celle des garçons. Si cet enseignement n'était alors probablement pas axé vers la formation de praticiennes des sciences, il demeure qu'après la mise en place des cercles - dont l'un des buts fut de susciter des vocations scientifiques $^{34}$ - les religieuses encouragèrent largement leurs élèves à s'engager dans le mouvement. Souvent poussées par les responsables de leur congrégation, les sœurs furent d'ailleurs nombreuses à assister aux cours de l'Institut botanique, en particulier à ceux de Marie-Victorin. Même s'il y eut des religieuses pour affirmer que les sciences naturelles n'avaient pas leur place dans l'éducation des jeunes filles ${ }^{35}$, l'étude des dossiers des cercles révèle un réel intérêt des filles pour les sciences, notamment chez les normaliennes. Certaines jeunes institutrices laïques exprimaient d'ailleurs leurs regrets, dans leurs lettres au secrétariat des CJN, de n'avoir pas eu la possibilité de poursuivre leurs études en sciences ${ }^{36}$.

Plus pragmatiquement, il faut remarquer aussi qu'au delà de l'intérêt pour les sciences, les CJN représentaient certainement, pour les filles, une diversion attrayante dans la routine des pensionnats. Ainsi, les excursions ont, semble-t-il, toujours constitué un élément très important dans la motivation des couventines habituellement confinées dans leur établissement ${ }^{37}$.

Quant au nombre de membres dans les cercles masculins, il resta relativement stable, autour de 2500 par année, durant à peu près toute la période étudiée. Compte tenu de la croissance démographique, cette stabilité correspond en fait à une régression nette de l'intérêt des garçons pour les CJN.

33. Concours organisé grâce à la SCHN, qui marque une étape importante dans la fondation des CJN, et où les filles ont raflé la plupart des premiers prix.

34. «Les CJN ont formé de véritables savants», affirme une responsable des CJN de la première heure, Marcelle Gauvreau, «ils en forment encore aujourd'hui pour le monde de demain» (La Presse, $1^{\text {er }}$ octobre 1956).

35. Certaines religieuses se plaignent, dans leurs lettres au secrétariat des CJN, d'être mal épaulées par leur hiérarchie; voir, entre autres, ACJN, 16P5b/1166.

36. Le courrier avec le secrétariat des CJN est souvent l'occasion de confidences de la part des responsables locaux des cercles; voir, entre autres, ACJN, 16P5/1568.

37. Entrevues avec sœur Hélène Fournier, snjm, et sœur Yvette Ravenelle, csc, anciennes responsables de CJN dans les années 1950 et 1960. 
Assez étonnante, cette situation s'explique de plusieurs façons: d'abord, il a déjà été noté que les œuvres congréganistes d'éducation masculines étaient numériquement moins importantes que celles de leurs homologues féminines. Ensuite, les congrégations de religieux paraissent n'avoir jamais entrepris, à la différence des communautés féminines, des programmes d'implantation systématique de cercles dans leurs établissements, du moins jamais sur la même échelle; au contraire, les frères sollicitaient le plus souvent individuellement le secrétariat des CJN en vue de fonder un cercle. Mais surtout, les communautés de religieux, qui enseignaient exclusivement à des garçons, offraient apparemment des choix d'activités plus séduisantes pour leurs élèves, tels que les sports, les corps de musique, de cadets, etc., et elles semblèrent manquer de volontaires pour les cercles. Comme le notait un Clerc de Saint-Viateur: «Quand on a pourvu toutes les activités scolaires d'effectifs convenables, il n'en reste plus pour celles d'un CJN qui s'inscrit au dernier rang de la liste d'emploi. C'est pourtant une discipline intellectuelle bien propre à enrichir un jeune esprit ${ }^{38} .{ }^{\prime \prime}$

\section{ÂGE DES MEMBRES, NIVEAUX SCOLAIRES ET TYPES D'ÉTABLISSEMENTS}

Ce n'est qu'après 1953 qu'il devient possible d'étudier les variations de la moyenne d'âge des membres, les feuilles de rapport n'en faisant que rarement mention avant. Par la suite, la question est, chaque année, éludée dans 15 à $20 \%$ des rapports. En ce qui concerne les niveaux scolaires, les données ne sont réellement utilisables qu'à partir de 1955 et, même après cette date, l'indication manque dans environ $35 \%$ des cas. Dans ce contexte, la présente étude n'apporte vraisemblablement ici qu'une indication de tendance.

Globalement, la moyenne d'âge s'établit, dans les années 1950, autour de 13 ans, un âge charnière entre les niveaux primaire et secondaire, pour graduellement descendre aux environs de 11,5 ans après 1966. Ce rajeunissement progressif semble indiquer que le déclin des cercles, dans les années 1960, entama d'abord les effectifs les plus âgés pour conserver les membres les plus jeunes. Cette moyenne d'âge varie également selon les congrégations, ce qui reflète leur clientèle. De plus, les garçons ont été, en moyenne, un peu plus âgés que les filles dans le mouvement. Pour leur part, les naturalistes du secteur laïque furent très jeunes (autour de 11 ans), se recrutant surtout au primaire. E128/3.

38. Lucien Charbonneau, csv, Rapport de 1948-1949 (Montréal, Archives des CSV), 
La répartition des cercles et des membres selon le niveau scolaire confirme, en outre, le rajeunissement des effectifs des CJN dans les années 1960, la position relative du primaire augmentant sensiblement durant cette décennie (55\% en 1966 contre $46 \%$ en 1956). Les CJN ne furent jamais très nombreux dans les collèges classiques (ils étaient présents dans à peu près la moitié des collèges classiques en 19601961); les effectifs des CJN dans ces institutions (qui rassemblaient autour de $1,7 \%$ de la population scolaire en 1960-1961) représentaient $6,7 \%$ de l'ensemble des membres des cercles en 1956; le niveau collégial n'en constituait plus que 3,6\% en 1966. La même tendance s'observe au niveau secondaire qui fléchit de $33 \%$ à $26 \%$ durant la même période. L'identification des divers types de maisons d'enseignement (repérés notamment grâce aux indications de l'adresse postale du cercle), semble confirmer la prépondérance des niveaux primaire et secondaire pour la période avant 1953.

Dans ce contexte, il n'est guère étonnant que la contribution des $\mathrm{CJN}$ à la recherche scientifique (en phytogéographie, en particulier) ait été à peu près nulle, la qualité des travaux, très inégale, reflétant assez directement le niveau scolaire - et la formation du directeur du cercle. Au reste, les $\mathrm{CJN}$ ont eu dès le début une orientation résolument pédagogique et ils n'ont jamais servi à «drainer [...] vers MarieVictorin» les éventuelles découvertes des jeunes naturalistes ${ }^{39}$. Même s'il salua, à une ou deux reprises dans Le Devoir, des petites trouvailles intéressantes des cercles, le directeur de l'Institut botanique n'a jamais fait d'efforts pour utiliser les cercles dans ce sens.

La place des écoles normales dans le mouvement attire l'attention. De fait, ces établissements ont été particulièrement visés (ciblés, dirait-on aujourd'hui) dès les années 1930, à cause de leur influence sur l'habitus - pour emprunter une notion propre à la sociologie de P. Bourdieu - des futurs enseignants. Les visites dans les écoles normales et les scolasticats apparaissent clairement comme des priorités dans les directives que la SCHN adressa au premier directeur des $\mathrm{CJN}$, le frère Adrien $^{40}$, et ce souci de rejoindre les enseignants pendant leur formation se retrouve régulièrement par la suite ${ }^{41}$. Là encore, les filles sont largement représentées: si, en 1956, un cercle fonctionnait dans 22 écoles normales de filles sur les cinquante-huit qui existaient dans la province, il ne s'en trouvait aucun dans les

39. Jean-Claude Guédon, loc. cit., 101-102.

40. Voir le rapport de la $3^{e}$ séance du Conseil de la SCHN, 31 mai 1935, archives de la SCHN, Service des archives de l'UQAM, 15P1/1.

41. Voir notamment Sœur Marie-Jean-Eudes, ssa, «L'enseignement de l'histoire naturelle à la normale», Sciences et aventures, 8,5 (mai 1952): 106-108. 
écoles normales de garçons ${ }^{42}$. En fait, les cercles étaient assez bien représentés dans tous les types de maisons d'enseignement assignées aux filles: ils n'étaient pas rares dans les écoles ménagères et, plus tard, dans les instituts familiaux; ils furent, de plus, relativement populaires dans les couvents et les pensionnats.

Par ailleurs, on remarque la présence des CJN dans les écoles rurales (école de rang) entre 1931 et 1950. Ces cercles tendent à correspondre aussi avec la poussée des CJN laïques entre 1936 et 1942 déjà constatée à la figure 2. Cet accroissement est assez bien délimité géographiquement (Beauce, Rimouski, Matapédia), de sorte qu'il est possible de conclure à une action concertée, peut-être initiée et encouragée par des inspecteurs d'écoles.

De plus, les bouleversements du système scolaire de la province dans les années 1960, la régionalisation de l'école secondaire, l'apparition des polyvalentes et des cégeps ont eu pour effet de faire disparaître les cercles; les CJN qui firent le saut vers les nouvelles structures furent très peu nombreux et, en général, cessèrent leurs activités peu de temps après.

Finalement, le cercle indépendant du système scolaire, le «CJNloisir», est toujours resté tout à fait marginal dans le mouvement (de 1931 à 1971,50 cercles sur 1959 , soit $2,5 \%)^{43}$. Il fut, en effet, extrêmement rare que des animateurs bénévoles tentent d'organiser un cercle dans leur entourage: les quelques tentatives qui ont laissé des traces se sont avérées le plus souvent sans lendemain. Dans les années 1970 , ce type de CJN s'est toutefois développé et constitue aujourd'hui l'essentiel des cercles.

\section{LA RÉPARTITION GÉOGRAPHIQUE DES CERCLES}

Notre compilation des cercles a permis de repérer assez précisément l'activité des CJN dans chaque comté, nonobstant les divers changements de la carte électorale de 1931 à 1971. Dans le but de simplifier les représentations graphiques, treize régions ont été arbitrairement déterminées pour lesquelles les figures 6 et 7 fournissent la progression des cercles pour les années 1946 et $1966^{44}$. Pratiquement jamais omise, cette donnée permet de préciser assez fidèlement l'aire de diffusion des cercles.

42. Voir Rapport du Congrès national sur les sciences naturelles (1956), 58, ACJN, $16 \mathrm{P} 12 \mathrm{a} / 8$.

43. Les «CJN-loisirs» regroupent une clientèle de jeunes. L'expérience des CNA, les cercles de naturalistes adultes (ou amateurs), dans les années 1950, sera encore plus limitée.

44. Le nombre de cercles a été ici retenu comme variable déterminante; le nombre de membres montre, à une exception près, les mêmes tendances. 


\section{FIGURES 5 ET 6}

Répartition des cercles par régions en 1946 et 1966

\section{6}

Nombre de cercles

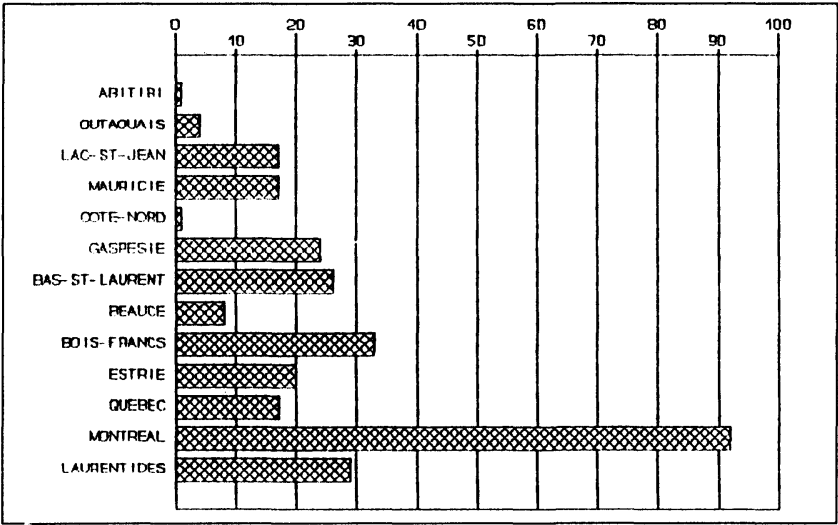

1966

Nombre de cercles

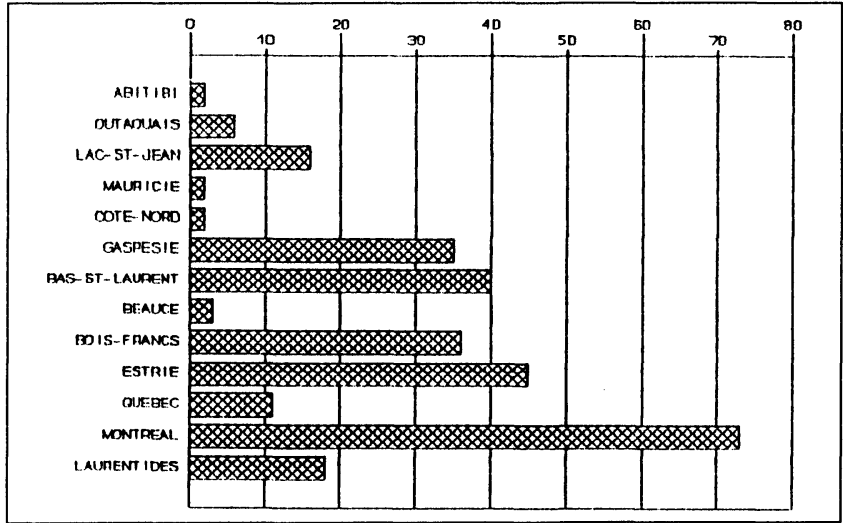

Source: ACJN, 16P5b/1-1959. 
Remarquons d'abord que la prépondérance de Montréal quant au nombre de cercles transparaît clairement jusqu'aux années 1960: de 1936 à 1961, la métropole rassembla régulièrement près d'un tiers des CJN. S'il est tentant d'y voir un effet de la concentration démographique, on note que Québec, deuxième centre urbain de la province, avec autour de $6 \%$ du nombre total de cercles durant la même période, resta, tout aussi régulièrement, en deçà de régions comme les Laurentides, la Gaspésie ou l'Estrie. En fait, des comtés tels que Rimouski, Matane ou Matapédia (fiefs des Sœurs du Saint-Rosaire) se comparèrent souvent favorablement avec Québec et c'est encore dans les régions périphériques (Gaspésie, Bas-Saint-Laurent, Lac-SaintJean) que se retrouvent une grande partie des CJN en 1993.

Par ailleurs, le déclin des CJN - aussi bien en termes de nombre de cercles que de nombre de membres - durant les années 1960 frappa d'abord la région montréalaise. De fait, à partir de 1961, plusieurs régions améliorèrent notablement leur position relative, quant au nombre de cercles, par rapport à Montréal qui fut dépassé au début des années 1970. Les causes de l'effondrement des CJN se seraient donc fait sentir avec un certain décalage à l'extérieur de la métropole.

En fait, cette répartition géographique des cercles révèle surtout la concentration du clergé régulier au Québec. Pour des raisons complexes, souvent liées aux circonstances de leur fondation ou de leur installation au Québec, les communautés étaient très inégalement réparties dans la province et se partagaient, en un subtil accommodement avec le clergé séculier, l'espace et les fonctions. C'est ainsi que, en gros, les Sœurs de Sainte-Anne se retrouvaient surtout dans la région montréalaise tout comme la Congrégation Notre-Dame; les Sœurs de Sainte-Croix étaient fortement implantées dans les Laurentides; les Sœurs de Saint-Joseph de Saint-Hyacinthe, dans les BoisFrancs et les Sœurs du Saint-Rosaire, dans le Bas-Saint-Laurent et la Gaspésie. Jusqu'à un certain point, le dynamisme d'une région reflétait en grande partie l'implantation et les efforts des religieux: les efforts directs d'abord, dans leurs établissements, mais peut-être aussi, d'une façon indirecte, leur influence sur les enseignants d'une région, à travers leurs écoles normales et leurs scolasticats, notamment.

Finalement, le développement des cercles à l'étranger, maintes fois mentionné, voire monté en épingle, durant les années 1930 et 1940, apparaît marginal; les CJN du Bengale, d'Alaska et du Japon se révéleront, si on se fie aux sources, des entreprises de courte durée et isolées, conduites par quelques missionnaires canadiens-français, mal épaulés par la direction des cercles. L'implantation des cercles en France, juste avant la guerre, laissa peu de traces et les quelques CJN 
du Maine, dépendant des Sœurs du Saint-Rosaire pour la plupart, représentent la seule expérience durable à l'extérieur du Québec.

\section{CONCLUSION}

Les CJN, qui célèbrent en 1996 leur soixante-cinquième anniversaire, ne se distinguent pratiquement plus aujourd'hui, sinon par leur histoire, des autres clubs scientifiques au Québec. Même si l'analyse des résultats reste ici succincte, cet exposé parvient cependant à montrer que les cercles se sont développés, avant les années 1970, presque exclusivement en milieu scolaire, constituant ainsi un cas hybride entre des classes de sciences naturelles régulières et les clubs scientifiques; dans ce sens, il est sans doute plus juste de parler, pour la période avant 1970, d'enseignement des sciences que de vulgarisation scientifique en ce qui concerne les CJN. De plus, la contribution des CJN à la recherche scientifique, en botanique notamment, est pratiquement inexistante; il est sans doute illusoire de penser que MarieVictorin ait pu sérieusement envisager de s'appuyer sur les cercles pour l'aider dans ses recherches en phytogéographie.

Cette recherche montre aussi que les cercles ont été d'abord et avant tout le fait des congrégations religieuses d'enseignement et qu'ils ont touché un public à la fois très jeune (surtout les niveaux primaire et secondaire) et très majoritairement féminin. À cet égard, les congrégations féminines se signalent tout particulièrement et c'est essentiellement sur elles que va reposer la croissance des CJN dès 1931. De fait, les caractéristiques des membres des cercles reflètent souvent directement les traits spécifiques de la clientèle de chaque congrégation et, partant, les efforts de ses sujets dans le mouvement des jeunes naturalistes. Ainsi, le sexe mais aussi l'âge des membres et la répartition géographique des cercles traduiront, en grande partie, le dynamisme de chaque communauté face aux CJN.

Les CJN peuvent donc apparaître comme le produit d'une collaboration un peu ambiguë entre certains scientifiques et les congrégations qui permit aux religieux d'améliorer l'image - et certainement aussi la qualité - de leur enseignement des sciences sans bouleverser leurs plans d'études. Sévèrement critiqué à la fin des années 1920, entre autres par Adrien Pouliot, l'enseignement des sciences au Québec changea peu; les CJN constituèrent même l'une des rares réponses du système éducatif aux multiples appels en faveur de réformes. Or, pour le frère Marie-Victorin, par exemple, il fallait «toucher les choses à leur base, c'est-à-dire dans le domaine même de l'éducation ${ }^{45}$ ». En fait, le projet des responsables de la SCHN et de

45. Marie-Victorin, loc. cit., 17. 
l'ACFAS fut sans commune mesure avec les objectifs, plus timorés, des œuvres congréganistes d'éducation.

L'intérêt des scientifiques pour les cercles s'émoussa graduellement à mesure que l'espace scientifique s'autonomisait, de sorte que, dès la fin des années 1940 et jusqu'au début des années 1970, les CJN allaient se maintenir presque exclusivement grâce aux congrégations d'enseignement. Après la laïcisation du système éducatif du Québec, le mouvement poursuivit son action en se rétractant et en se stabilisant autour d'un petit noyau d'animateurs bénévoles.

$\mathrm{Du}$ fait des caractéristiques des membres des CJN, il est donc difficile d'assimiler, au sens strict, les cercles au «scientistic movement» décrit par J. Ben-David: les membres des cercles sont, dans l'ensemble, beaucoup trop jeunes pour avoir constitué ce groupe cohérent, militant, «who believe in science (...) as a valid way to truth and to effective mastery over nature as well as to the solution of the problems of the individual and his society ${ }^{46} \gg$. De plus, ils n'ont jamais, comme tels, influé directement sur le développement des sciences au Québec. L'impuissance des CJN, comme organisation, est notamment manifeste en ce qui concerne leur incapacité à obtenir du gouvernement provincial autre chose qu'une maigre allocation de subsistance plus ou moins régulière. Par ailleurs, les membres des cercles ne se recrutent pas particulièrement dans ces strates montantes et dynamiques qui forment le moteur du schéma de Ben-David, mais reflètent plutôt les caractéristiques spécifiques des clientèles de chaque congrégation.

Il faut noter cependant qu'en renouvelant même très partiellement l'enseignement scientifique, les CJN ont peut-être eu, finalement, un certain impact sur la perception des sciences au Québec. En ce sens, les CJN n'ont sans doute constitué qu'une sorte de mouvement scientifique par procuration. En effet, les responsables de la SCHN et de l'ACFAS, profitant du flou qui entourait les cercles, ont cherché à utiliser les CJN comme caution pour atteindre leurs objectifs. Il reste qu'il ne serait probablement pas facile de démontrer la part de l'influence des CJN sur un projet comme le Jardin botanique de Montréal, par exemple, ou sur la perception des sciences en général. 